\title{
Change Point Detection of EEG Signals Based on Particle Swarm Optimization
}

\author{
M.F. Mohamed Saaid1, W.A.B. Wan Abas1, H. Aroff2, N. Mokhtar2, R. Ramli1, and Z. Ibrahim3 \\ ${ }_{1}$ Department of Biomedical Engineering, Faculty of Enginnering, University of Malaya, Kuala Lumpur, Malaysia \\ 2 Department of Electrical Engineering, Faculty of Enginnering, University of Malaya, Kuala Lumpur, Malaysia \\ 3 Department of Mechatronics and Robotics, Center for Artificial Intelligence and Robotics (CAIRO) \\ Faculty of Electrical Engineering Universiti Teknologi Malaysia, Johor Bharu, Malaysia
}

INTRODUCTION

These Electroencephalography (EEG) is the variations of electrical fields in the cortex or on the surface of scalp caused by the physiological activities of the brain. Over the years, there have been many modern methods such as Computed Tomography (CT), Magnetic Resonance Imaging

(MRI), etc. However, electroencephalogram (EEG) signal is still play a very important role in the diagnosis of brain function [1]. The EEG provides high temporal resolution with millisecond precision [2]. It has been implemented in diagnosing epilepsy [3], brain injury [4], and various sleep disorders [5]. Recently, a Brain Computer Interface $(\mathrm{BCI})$ is developed, which translate EEG signal to actuate a device [6].

Change point detection is a classical problem in signal processing, where this tool help us to decide whether such a change occurred in the characteristics of the considered signal [7]. Various application of change point detections have been applied in EEG signal processing. Early warning system of an epileptic seizure could give a more time to treat the patients [8].The detection algorithm can also be used to segment different sleep stages measured by EEG method [9]. A common practice in time series analysis is to assume that time-varying structure of time series can be well estimated by a piecewise stationary process [10], 
which could reduce the complexity of finding the change

point.

In earlier work by Adak [10], Tree-based Adaptive Segmented Spectrogram algorithm (TASS) was proposed by recursively dividing the signals into two short segments, and the signal from the left side is compared with the right side of the segmented signals. One limitation of the Adak method is that it is not easily extended to multivariate data.

Davis et al. [11] proposed the Auto-PARM which used the minimum description length (MDL) principle. The AutoPARM finds the "best" combination of the number of breakpoints, their locations and AR orders of all stationary segments by minimizing the code length which is computed according to general coding rules. Genetic Algorithm (GA) was implemented to solve the MDL optimization problem.

Ombao et al. [12] proposed a smoothed localized complex exponentials (SLEX), where the time series is fitted into the orthogonal basis function. Like Adak, the SLEX model treats a series as piecewise stationary, where the pieces are determined by the choice of basis functions.

Recently in 2008, Last et al [13] develop a method for detecting abrupt changes to the time-varying power spectrum of a series, assuming that the series is locally stationary between change-points. However, he claimed that the process is computationally expensive. For real-time, he proposed that computing power spectrum can be done parallelized, independently of any window. Later, he showed the problem of using fixed window-design, where some of the true change point maybe not detected.

In this paper, we propose a change point detection algorithm, where the EEG signal is model by the sinusoidal function with a Heaviside function. The parameter of this model was estimated by using the least square approach, 
which the Particle Swarm Optimization (PSO) [14] is used to find all the parameter in the model, including the change point location. In this paper, the proposed approach was use to detect only a single change in the EEG signal in offline manner.

Full text is available at :

http://link.springer.com/chapter/10.1007/978-3-642-21729-6 122 